\title{
Tight Exponential Upper Bounds on the ML Decoding Error Probability of Block Codes Over Fully Interleaved Fading Channels *
}

\author{
I. Sason ${ }^{\dagger} \quad$ S. Shamai ${ }^{\ddagger} \quad$ D. Divsalar $\S$
}

August 28, 2003

\begin{abstract}
We derive in this paper tight exponential upper bounds on the decoding error probability of block codes which are operating over fully interleaved Rician fading channels, coherently detected and maximum-likelihood decoded. It is assumed that the fading samples are statistically independent and that perfect estimates of these samples are provided to the decoder. These upper bounds on the bit and block error probabilities are based on certain variations of the Gallager bounds. These bounds do not require integration in their final version and they are reasonably tight in a certain portion of the rate region exceeding the cutoff rate of the channel. By inserting inter-connections between these bounds, we show that they are generalized versions of some reported bounds for the binary-input AWGN channel.
\end{abstract}

Index Terms - Block codes, bounds, distance spectrum, fading channels, ML decoding, uniform interleaver.

${ }^{*}$ The paper appears in the IEEE Trans. on Communications, vol. 51, no. 8, pp. 1296-1305, August 2003. The paper was submitted on March 2002, and revised on December 2002. This work was presented in part at the Sixth International Symposium on Communication Theory and Applications (ISCTA 06), Ambleside, UK, July 15-20, 2001.

${ }^{\dagger}$ Department of Computer Science and Communications, EPFL - Swiss Federal Institute of Technology, Lausanne 1015, Switzerland, e-mail: igal.sason@epfl.ch. Part of this work was done while the author was with the Department of Electrical Engineering, Technion-Israel Institute of Technology, Haifa, Israel.

${ }^{\ddagger}$ Department of Electrical Engineering, Technion-Israel Institute of Technology, Haifa 32000, Israel, e-mail: sshlomo@ee.technion.ac.il.

${ }^{\S}$ Sequoia Communications, Los Angeles, and Jet Propulsion Laboratory, Pasadena, CA 91109, USA, e-mail: dariush@shannon.jpl.nasa.gov. 


\section{Introduction}

In recent years there has been renewed interest in deriving tight bounds on the error performance of specific codes and ensembles, based on their distance spectrum. The incentive for introducing and applying such bounds has increased with the introduction of turbo codes [1], the rediscovery of lowdensity parity-check codes [2], and the introduction of efficiently decoded ensembles of turbo-like codes (e.g, [3]). Clearly, the desired bounds must not be subject to the union bound limitation, since for long blocks, these ensembles of turbo-like codes perform reliably at rates which are considerably above the cutoff rate of the channel (recalling that union bounds for long codes are not informative at the portion of the rate region above the cutoff rate of the channel, where the performance of these capacity-approaching codes is most appealing). Although maximum-likelihood (ML) decoding is in general prohibitively complex for long enough block codes, the derivation of upper bounds on the ML decoding error probability is of interest, providing an ultimate indication of the system performance. Further, the structure of efficient codes is usually not available, necessitating efficient bounds to rely only on basic features, such as the distance spectrum or input-output weight enumeration function (IOWEF) of the examined codes or ensembles. These latter features can usually be found by some analytical methods (see e.g., $[4,5,6])$.

In this paper, we derive simple and tight bounds on the decoding error probability of block codes which are operating over fully interleaved Rician fading channels, coherently detected and ML decoded. These upper bounds are based on certain variations of Gallager bounds. The bounds are simple, since they do not require any integration in their final version (as opposed to other bounds, e.g., [7]-[11]). They are also tight in a portion of the rate region above the cutoff rate of the channel. Certain interconnections between these bounds are demonstrated, and we show that the bounds which are introduced in this paper form a generalization of some reported bounds for the binary-input AWGN channel $[12,13,14]$.

The generalization of the Viterbi \& Viterbi bound [14] for fully interleaved fading channels with perfect channel side information (CSI), as reported in [15], seems to be imprecise. Our reservation stems from the fact that the Viterbi \& Viterbi bounding technique [14] is invalidated once the parameter $H=\exp \left(-\frac{E_{s}}{N_{0}}\right)$ (in the AWGN case) is replaced by $H$ in [15], Eq. (12), for the fast Rician fading channel. The specific correlations in [14] demand special care when generalized to fading channels. A generalization of the Viterbi \& Viterbi bound for imperfect CSI is considered 
in [16]. Again, this is problematic as the technique relies on the arguments of [15], when now $H$ is replaced by the one corresponding to imperfect CSI.

In this paper we suggest an alternative generalization of the Viterbi \& Viterbi upper bound [14] for fully interleaved fading channels. Throughout the paper, we assume perfect CSI for the realizations of the i.i.d. fading values. The analysis via tight upper bounds on the ML decoding error probability for fully interleaved fading channels with imperfect CSI and their applications to turbo and turbo-like codes was recently studied in [7, 11].

The paper is organized as follows: the derivation of an upper bound on the ML decoding error probability for fully interleaved fading channels with perfect CSI is introduced in Section 2.1. It is demonstrated that by determining the parameters of this bound in an appropriate way, a generalization of the Viterbi \& Viterbi upper bound for fully interleaved fading channels is obtained. This generalized bound is derived in the appendix, and it is demonstrated in Section 2.2 that it can be derived as a particular case of the upper bound in Section 2.1. It is shown in Section 2.3 that the first version of the Duman and Salehi upper bound for a binary-input AWGN channel (see [12]) is a particular case of the upper bound in Section 2.1, where the latter bound is applicable for a general fully interleaved Rician fading channel. Optimizations of the parameters of the upper bound in Section 2.1 are discussed in Section 2.4. Inter-connections between these bounds are inserted in Section 2.5, based on their geometrical interpretation. An application of these bounds for the ensemble of the uniformly interleaved repeat and accumulate codes [3] over fully interleaved fading channels is exemplified in Section 3, and various upper bounds on the decoding error probability are compared there. We finally conclude our discussion in Section 4.

\section{Analysis and Discussion}

\subsection{Derivation of the upper bound}

In this section we derive an upper bound on the ML decoding error probability of binary and linear block codes operating over fully interleaved Rician fading channels.

Consider a binary and linear $(n, k)$ block code $C$ of rate $R=\frac{k}{n}$ bits per channel use, and suppose that its distance spectrum $\left\{S_{d}\right\}_{d=0}^{n}$ is calculable (numerically or analytically). Suppose the code is BPSK modulated and transmitted over a fully interleaved Rician fading channel, with a perfect CSI 
of the i.i.d. fading samples at the receiver. Here $E_{s}=R E_{b}$, where $E_{s}$ and $E_{b}$ are the energies per coded symbol and per information bit, respectively. The following equality holds for the conditional probability density functions $(p d f)$ with binary inputs: $p_{0}(y, a)=p_{1}(-y, a)$, where $y=a x+\nu$ is the received signal corresponding to antipodal signaling $x \in\left\{-\sqrt{E_{s}},+\sqrt{E_{s}}\right\}$, i.i.d. fading samples $(a)$ and additive white Gaussian noise $(\nu)$. We denote the $p d f$ of the i.i.d. fading samples $(a)$ by $p(\cdot)$ (the effect of the phases of the fading measurements is eliminated at the coherent receiver, and the amplitudes of the fades during each symbol are treated as non-negative random variables). Clearly, the fading realization $(a)$ which is ideally provided to the receiver, is interpreted as part of the measurements and is independent of the transmitted signal. It follows that

$$
p_{0}(y, a)=p_{1}(-y, a)=\frac{1}{\sqrt{2 \pi}} \exp \left[-\frac{\left(y-a \sqrt{2 E_{s} / N_{0}}\right)^{2}}{2}\right] \cdot p(a)
$$

where $-\infty<y<+\infty$ and $a \geq 0$, assuming that the observation $y$ is already normalized such that the additive noise has unit variance.

Let the block code $C$ be partitioned into a set of subcodes $\left\{C_{d}\right\}_{d=1}^{n}$, where every subcode $C_{d}$ includes all the codewords possessing a constant Hamming weight $d(d=1,2 \ldots n)$, and the allzero codeword. Let $P_{e}(d)$ denote the conditional block error probability of the subcode $C_{d}$ under ML decoding, where we assume that the all-zero codeword is the transmitted codeword (since the binary block code $C$ is linear and we consider a memoryless binary-input output-symmetric channel, this assumption incurs no loss of generality). Based on the union bound, we obtain an upper bound on the decoding error probability of the binary, linear block code $C$ under ML decoding

$$
P_{e} \leq \sum_{d=d_{\min }}^{d_{\max }} P_{e}(d)
$$

where $d_{\min }$ and $d_{\max }$ denote the minimal and maximal Hamming weights of the nonzero codewords of the code $C$, respectively. The generalization of the second version of Duman \& Salehi bounds (i.e., the generalization of the DS2 bound in [11]), which relies on the Gallager bounding technique yields the following upper bound on $P_{e}(d)$

$$
\begin{aligned}
P_{e}(d) \leq\left(S_{d}\right)^{\rho}\{ & \left(\int_{-\infty}^{+\infty} \int_{0}^{\infty} \psi(y, a)^{1-\frac{1}{\rho}} p_{0}(y, a)^{\frac{1}{\rho}} d a d y\right)^{(1-\delta) \rho} . \\
& \left.\left(\int_{-\infty}^{+\infty} \int_{0}^{\infty} \psi(y, a)^{1-\frac{1}{\rho}} p_{0}(y, a)^{\frac{1}{\rho}-\lambda} p_{1}(y, a)^{\lambda} d a d y\right)^{\delta \rho}\right\}^{n},
\end{aligned}
$$


where $d$ is the Hamming weight of the non-zero codewords in the subcode $C_{d}, \delta \triangleq \frac{d}{n}$ designates the normalized Hamming weight of these codewords $(0 \leq \delta \leq 1), 0 \leq \rho \leq 1, \lambda \geq 0$, and $\psi(\cdot, \cdot)$ is an arbitrary joint pdf of the measurements $y, a$.

In contrast to [10], where the optimal function $\psi$ (which can be viewed as a tilting measure with respect to the two measurements $y$ and $a$ ) was pursued, here for the sake of closed form expressions, only an exponential tilting measure is examined. Let $\psi(\cdot, \cdot)$ be the following $p d f$ :

$$
\psi(y, a)=\frac{\sqrt{\frac{\alpha}{2 \pi}} \exp \left[-\frac{\alpha}{2}\left(y-a u \sqrt{\frac{2 E_{s}}{N_{0}}}\right)^{2}-\frac{\alpha v^{2} a^{2} E_{s}}{N_{0}}\right] \cdot p(a)}{\int_{0}^{\infty} p(a) \cdot \exp \left(-\frac{\alpha v^{2} a^{2} E_{s}}{N_{0}}\right) d a}, \quad-\infty<y<+\infty
$$

where $\alpha$ is an arbitrary non-negative number and $u, v$ are arbitrary real numbers.

We assume here that the fading amplitude $(a)$ during each symbol is Rician distributed, so $p(\cdot)$ admits the form

$$
p(a)=2(1+K) a e^{-(1+K) a^{2}-K} I_{0}(2 a \sqrt{K(K+1)}), \quad a \geq 0,
$$

where the Rician parameter $K$ stands for the power ratio of the direct to the diffused received paths.

Let $c$ denote the normalization factor appearing in the denominator of $\psi(\cdot, \cdot)$ in $(4)$ :

$$
c=\int_{0}^{\infty} p(a) \cdot \exp \left(-\frac{\alpha v^{2} E_{s}}{N_{0}} \cdot a^{2}\right) d a=E\left[\exp \left(-a^{2} t\right)\right]
$$

where $t=\frac{\alpha v^{2} E_{s}}{N_{0}}$ and $E$ stands for the statistical expectation (with respect to $a$ ). According to the Rician distribution of $a$, one obtains that

$$
c=\frac{1+K}{1+K+t} \cdot \exp \left(-\frac{K t}{1+K+t}\right), \quad \text { if } \quad 1+K+t>0 .
$$

The condition $1+K+t>0$ holds since we assume that $\alpha \geq 0$ (which therefore yields that $t \geq 0$ ), and clearly $K \geq 0$. A calculation of the integrals which are involved in the upper bound (3) with the $\operatorname{pdf} \psi(\cdot, \cdot)$ in $(4)$ gives

$$
\int_{-\infty}^{+\infty} \int_{0}^{\infty} \psi(y, a)^{1-\frac{1}{\rho}} p_{0}(y, a)^{\frac{1}{\rho}} d a d y=\frac{c^{\frac{1}{\rho}-1} \alpha^{\frac{1}{2}\left(1-\frac{1}{\rho}\right)}}{\sqrt{\alpha-\frac{\alpha-1}{\rho}}} \cdot \frac{1+K}{1+K+\nu} \cdot \exp \left(-\frac{K \nu}{1+K+\nu}\right)
$$


where

$$
\nu=\left[\alpha\left(u^{2}+v^{2}\right)-\frac{\alpha\left(u^{2}+v^{2}\right)-1}{\rho}-\left(\alpha-\frac{\alpha-1}{\rho}\right)^{-1}\left(\alpha u-\frac{\alpha u-1}{\rho}\right)^{2}\right] \cdot \frac{E_{s}}{N_{0}},
$$

under the assumption that $1+K+\nu>0$.

A calculation of the second integral in (3) yields:

$\int_{-\infty}^{+\infty} \int_{0}^{\infty} \psi(y, a)^{1-\frac{1}{\rho}} p_{0}(y, a)^{\frac{1}{\rho}-\lambda} p_{1}(y, a)^{\lambda} d a d y=\frac{c^{\frac{1}{\rho}-1} \alpha^{\frac{1}{2}\left(1-\frac{1}{\rho}\right)}}{\sqrt{\alpha-\frac{\alpha-1}{\rho}}} \cdot \frac{1+K}{1+K+\varepsilon} \cdot \exp \left(-\frac{K \varepsilon}{1+K+\varepsilon}\right)$

where

$$
\varepsilon=\left[\alpha\left(u^{2}+v^{2}\right)-\frac{\alpha\left(u^{2}+v^{2}\right)-1}{\rho}-\frac{\left(\alpha u-\frac{\alpha u-1}{\rho}-2 \lambda\right)^{2}}{\alpha-\frac{\alpha-1}{\rho}}\right] \cdot \frac{E_{s}}{N_{0}},
$$

under the assumption that $1+K+\varepsilon>0$.

By substituting (6) and (8) in the upper bound on $P_{e}(d)(3)$, one obtains an upper bound on the conditional ML decoding error probability of the subcode $C_{d}$ (under the assumption that the all-zero codeword is transmitted):

$$
\begin{aligned}
P_{e}(d) \leq & \left(S_{d}\right)^{\rho} \alpha^{-\frac{n(1-\rho)}{2}}\left(\alpha-\frac{\alpha-1}{\rho}\right)^{-\frac{n \rho}{2}} \\
& \cdot\left(\frac{1+K}{1+K+t}\right)^{n(1-\rho)} \cdot \exp \left(-\frac{n(1-\rho) K t}{1+K+t}\right) \\
& \cdot\left(\frac{1+K}{1+K+\varepsilon}\right)^{d \rho} \cdot \exp \left(-\frac{K \varepsilon d \rho}{1+K+\varepsilon}\right) \\
& \cdot\left(\frac{1+K}{1+K+\nu}\right)^{(n-d) \rho} \cdot \exp \left(-\frac{K \nu(n-d) \rho}{1+K+\nu}\right),
\end{aligned}
$$

where $t, \nu, \varepsilon$ are introduced in (5), (7) and (9) respectively. The parameters above lie in the regions: $0 \leq \alpha<\frac{1}{1-\rho}$ (since $\alpha$ should be non-negative and also $\left.\alpha-\frac{\alpha-1}{\rho}>0\right), 0<\rho \leq 1, \lambda>0$ and $u, v$ are arbitrary real numbers. In order to get the tightest upper bound within this family, these parameters should be optimized. 
Following the arguments in [10], a similar upper bound on the bit error probability is derived for binary and linear block codes. To that end, one relies on the input-output weight distribution of the examined code or ensemble (the reader is referred to $[4,5,6]$ which derive several techniques for calculating the input-output weight distribution of codes and ensembles). In the final form of the upper bound on the bit error probability under ML decoding, the distance spectrum $\left\{S_{d}\right\}_{d=0}^{n}$ which appears in (3) is replaced by $S_{d}^{\prime}=\sum_{w=1}^{k}\left\{\left(\frac{w}{k}\right) A_{w, d}\right\}$. Here, $A_{w, d}$ designates the number of codewords which are encoded by information bits of Hamming weight $w$ and whose total Hamming weight (after the encoding) is $d$ (where $0 \leq w \leq k$ and $0 \leq d \leq n$ ).

In the following subsections we show that the upper bound (10) yields a generalization of the Viterbi \& Viterbi bound [14] and the first version of the Duman and Salehi bounds [12], where the latter two bounds apply to the binary-input AWGN channel. An alternative generalization of the Viterbi \& Viterbi bound for fully interleaved Rician fading channels (with perfect CSI at the receiver) is derived in the Appendix.

\subsection{Generalization of the Viterbi \& Viterbi bound [14] for fully interleaved Rician fading channels}

The Viterbi \& Viterbi bound is an upper bound on the ML decoding error probability of binary linear block codes operating over a binary-input AWGN channel [14]. A generalization of this bound for fully interleaved Rician fading channels with perfect CSI at the receiver is derived in the Appendix of this paper. It admits the form (2), where the upper bound on $P_{e}(d)$ is

$$
\begin{aligned}
P_{e}(d) \leq\left(S_{d}\right)^{\rho} & \left(\frac{1+K}{1+K+\beta_{3}}\right)^{n(1-\rho)} \cdot \exp \left(-\frac{K \beta_{3} n(1-\rho)}{1+K+\beta_{3}}\right) \\
& \cdot\left(\frac{1+K}{1+K+\beta_{1}}\right)^{d \rho} \cdot \exp \left(-\frac{K \beta_{1} d \rho}{1+K+\beta_{1}}\right) \\
& \cdot\left(\frac{1+K}{1+K+\beta_{2}}\right)^{(n-d) \rho} \cdot \exp \left(-\frac{K \beta_{2}(n-d) \rho}{1+K+\beta_{2}}\right),
\end{aligned}
$$

and

$$
0 \leq \rho \leq 1, \quad \beta_{1}=\frac{E_{s}}{N_{0}}, \quad \beta_{2}=\frac{E_{s}}{N_{0}} \cdot\left[1-(1+\zeta(1-\rho))^{2}\right], \quad \beta_{3}=\frac{E_{s}}{N_{0}} \cdot\left[1-(1-\zeta \rho)^{2}\right] .
$$


By comparing the upper bounds (10) and (11), we want to determine the parameters in (10) so that the upper bound (11) results as a particular case of (10). To this end, we set $\alpha=1$ in (10), and then we try to choose the remaining parameters in (10) so that the following equalities hold:

$$
\beta_{1}=\varepsilon, \quad \beta_{2}=\nu, \quad \beta_{3}=t .
$$

Based on Eqs. (5), (10) and (11), straightforward algebra reveals that the bound (10) specializes to the generalized Viterbi \& Viterbi bound (11), by setting the parameters in (10) as follows:

$$
\alpha=1, \quad u=1-\zeta \rho, \quad v=\sqrt{1-(1-\zeta \rho)^{2}}, \quad \lambda=\frac{1+\zeta(1-\rho)}{2}
$$

in case that $\beta_{3} \geq 0$ in (11). However, the optimal value of $\beta_{3}$ in terms of minimizing the upper bound (11) is non-negative. To see this, we rewrite the upper bound (11) in the form:

$$
P_{e}(d) \leq \exp (-n E)
$$

where

$$
\begin{aligned}
E & =\frac{K \beta_{1} \delta \rho}{1+K+\beta_{1}}+\frac{K \beta_{2}(1-\delta) \rho}{1+K+\beta_{2}}+\frac{K \beta_{3}(1-\rho)}{1+K+\beta_{3}}-\rho \mathrm{r}(\delta) \\
& +\delta \rho \ln \left(1+\frac{\beta_{1}}{1+K}\right)+(1-\delta) \rho \ln \left(1+\frac{\beta_{2}}{1+K}\right)+(1-\rho) \ln \left(1+\frac{\beta_{3}}{1+K}\right),
\end{aligned}
$$

and $\delta \triangleq \frac{d}{n}, \mathrm{r}(\delta) \triangleq \frac{\ln S_{d}}{n}$. By nulling the partial derivative of the error exponent $E$ with respect to $\zeta$ (recall that only $\beta_{2}$ and $\beta_{3}$ depend on $\zeta$ ), it can be verified that $0 \leq \zeta \rho \leq 1$, which yields from (12) that $\beta_{3} \geq 0$. We note that for the particular case of a binary-input AWGN channel, it follows from

(12) and (A.39) that $\beta_{3}=1-\left(\frac{1-\delta}{1-\delta+\delta \rho}\right)^{2}$. For a general fully interleaved Rician fading channel, the optimal value of $\beta_{3}$ does not admit a closed form expression, but it can be verified analytically that it is indeed non-negative. This observation makes the discussion in the case where $\beta_{3}$ is negative irrelevant, and therefore the generalization of the Viterbi \& Viterbi bound (11) (which is detailed in the Appendix) is a particular case of the upper bound (10) in Section 2.1.

\subsection{The first version of the Duman and Salehi bounds [12]}

We demonstrate here that the first version of the Duman and Salehi bounds, which is derived in [12] for a binary-input AWGN channel is also a particular case of the upper bound (10) in Section 2.1. 
To this end, we first write (10) in the limit case where $K \rightarrow \infty$ (as for an AWGN channel, the Rician factor tends to infinity), and obtain the upper bound:

$$
P_{e}(d) \leq\left(S_{d}\right)^{\rho}\left(\alpha-\frac{\alpha-1}{\rho}\right)^{-\frac{n \rho}{2}} \cdot \alpha^{-\frac{n(1-\rho)}{2}} \cdot \exp (-n(1-\rho) t) \cdot \exp (-\varepsilon d \rho) \exp (-\nu(n-d) \rho)
$$

By setting $v=0$, we get $t=0$ (based on the definition of the parameter $t$ before Eq. (5)), which reduces the upper bound (15) to the form:

$$
P_{e}(d) \leq\left(S_{d}\right)^{\rho} \alpha^{-\frac{n(1-\rho)}{2}}\left(\alpha-\frac{\alpha-1}{\rho}\right)^{-\frac{n \rho}{2}} \cdot \exp (-(\varepsilon-\nu) d \rho) \cdot \exp (-\nu n \rho) .
$$

The substitution of $\nu$ and $\varepsilon$ in (7) and (9) respectively, and the introduction of a new parameter $\beta$, where $\beta=\alpha u$ leads to the upper bound:

$$
\begin{aligned}
P_{e}(d) & \leq\left(S_{d}\right)^{\rho} \alpha^{-\frac{n(1-\rho)}{2}}\left(\alpha-\frac{\alpha-1}{\rho}\right)^{-\frac{n \rho}{2}} \\
& \cdot \exp \left[\left(-1+\frac{\beta^{2}}{\alpha} \cdot(1-\rho)+\frac{\rho\left(\beta-\frac{\beta-1}{\rho}\right)^{2}}{\alpha-\frac{\alpha-1}{\rho}}\right) \frac{n E_{s}}{N_{0}}\right] \\
& \cdot \exp \left[-\frac{4 \lambda \rho\left(\beta-\lambda-\frac{\beta-1}{\rho}\right)}{\alpha-\frac{\alpha-1}{\rho}} \frac{d E_{s}}{N_{0}}\right] .
\end{aligned}
$$

The latter bound coincides with the upper bound (15) in [12], which proves that (10) yields the first version of the Duman \& Salehi bounds in [12] as a particular case.

\subsection{Optimization of the parameters in the upper bound (10)}

We wish to reduce the number of parameters which have to be numerically optimized in the upper bound (10), as to get the tightest bound within this family. We first optimize the parameter $\lambda$ in the upper bound (10), and define for that aim a function $f_{1}$ which includes the terms in (10) which depend on $\lambda$ :

$$
f_{1}=\left(\frac{1+K}{1+K+\varepsilon}\right)^{d \rho} \cdot \exp \left(-\frac{K d \rho \varepsilon}{1+K+\varepsilon}\right)
$$

A short calculation reveals that

$$
\frac{\partial}{\partial \lambda}\left\{\ln \left(f_{1}\right)\right\}=\frac{4 d \rho}{\alpha-\frac{\alpha-1}{\rho}} \cdot \frac{(1+K)^{2}+\varepsilon}{(1+K+\varepsilon)^{2}} \cdot\left(\alpha u-\frac{\alpha u-1}{\rho}-2 \lambda\right) \cdot \frac{E_{s}}{N_{0}}
$$


which yields that the optimal value of $\lambda$ is

$$
\lambda=\left\{\begin{array}{ll}
\frac{1}{2}\left(\alpha u-\frac{\alpha u-1}{\rho}\right) & \text { if } \alpha u<\frac{1}{1-\rho} \\
0 & \text { otherwise }
\end{array} .\right.
$$

The choice $\lambda=0$ in (10) provides a useless upper bound on the decoding error probability (this choice in Gallager's bound already gives an upper bound which equals to unity, and by invoking Jensen's bound for the derivation of (10), one can only loosen this upper bound). Therefore, without any loss of generality, one can restrict the optimization of (10) to the case where $\alpha u<\frac{1}{1-\rho}$, and choose the parameter $\lambda$ under this restriction to be

$$
\lambda=\frac{1}{2}\left(\alpha u-\frac{\alpha u-1}{\rho}\right) .
$$

The substitution of (18) in (9) and a simplification of (9) give

$$
\varepsilon=\left[\alpha\left(u^{2}+v^{2}\right)\left(1-\frac{1}{\rho}\right)+\frac{1}{\rho}\right] \frac{E_{s}}{N_{0}}, \quad \nu=\left(1-\frac{1}{\rho}\right)\left[\alpha v^{2}+\frac{\alpha(u-1)^{2}}{1-(1-\rho) \alpha}\right] \frac{E_{s}}{N_{0}} .
$$

The closed form expression (18) for the optimized parameter $\lambda$ reduces the number of parameters which should be optimized in (10) to four (namely, to optimizing $(\alpha, u, v, \rho)$ ).

We optimize now the upper bound (10) with respect to the parameter $r \triangleq \alpha v^{2}$ : Based on (5), (19) and the introduction of the new parameter $r$ (instead of the parameter $v$ ), we optionally express $t, \varepsilon, \nu$ as functions of $r$ :

$$
t=\frac{r E_{s}}{N_{0}}, \quad \nu=\left(1-\frac{1}{\rho}\right)\left[r+\frac{\alpha(u-1)^{2}}{1-\alpha(1-\rho)}\right] \frac{E_{s}}{N_{0}}, \quad \varepsilon=\left(1-\frac{1}{\rho}\right)\left(r+\alpha u^{2}-\frac{1}{1-\rho}\right) \frac{E_{s}}{N_{0}}
$$

where $r$ is a non-negative parameter (since $\alpha>0$ ). We optimize here $r$ by minimizing the relevant part of (10) which depends on $r$, and for this purpose, we introduce the following function:

$$
\begin{aligned}
f_{2}(r) & =\left(\frac{1+K}{1+K+t}\right)^{n(1-\rho)} \exp \left(-\frac{n(1-\rho) K t}{1+K+t}\right) \\
& \cdot\left(\frac{1+K}{1+K+\varepsilon}\right)^{d \rho} \exp \left(-\frac{K \varepsilon d \rho}{1+K+\varepsilon}\right) \\
& \cdot\left(\frac{1+K}{1+K+\nu}\right)^{(n-d) \rho} \exp \left(-\frac{K \nu(n-d) \rho}{1+K+\nu}\right) .
\end{aligned}
$$


A short calculation shows that

$$
\frac{\partial\left\{\ln \left(f_{2}\right)\right\}}{\partial r}=-n(1-\rho) \cdot \frac{E_{s}}{N_{0}}\left\{\frac{(1+K)^{2}+t}{(1+K+t)^{2}}-\frac{\delta\left[(1+K)^{2}+\varepsilon\right]}{(1+K+\varepsilon)^{2}}-\frac{(1-\delta)\left[(1+K)^{2}+\nu\right]}{(1+K+\nu)^{2}}\right\}
$$

where $\delta \triangleq \frac{d}{n}$ stands for the normalized Hamming weight. Nulling the partial derivative above gives the equation:

$$
\frac{(1+K)^{2}+t}{(1+K+t)^{2}}-\frac{\delta\left[(1+K)^{2}+\varepsilon\right]}{(1+K+\varepsilon)^{2}}-\frac{(1-\delta)\left[(1+K)^{2}+\nu\right]}{(1+K+\nu)^{2}}=0
$$

In general (i.e., for an arbitrary Rician factor $K \geq 0$ ), since $t, \nu, \varepsilon$ in (20) are linearly proportional to the parameter $r$, then (21) can be expressed as a polynomial equation of the fifth degree with the variable $r$. Therefore, for any selection of the three parameters $\alpha, u, \rho$ in (20) (where $0 \leq$ $\rho \leq 1, \alpha u<\frac{1}{1-\rho}$ and $0<\alpha<\frac{1}{1-\rho}$ ), the (non-negative) optimal value of $r$ can be numerically determined as a solution of the polynomial equation above. In particular, if $K=0$ (i.e., a Rayleigh fading channel), (21) is further simplified and admits the form:

$$
\frac{1}{1+t}-\frac{\delta}{1+\varepsilon}-\frac{1-\delta}{1-\nu}=0
$$

which easily transforms to a quadratic polynomial equation in $r$ (based on (20), which relates the parameters $\varepsilon, \nu, t$ to $r$ ). To conclude, for a fully interleaved Rician fading channel, one needs to solve numerically a polynomial equation in $r$ of the fifth degree, while for the particular case of a fully interleaved Rayleigh fading channel, this equation is simplified to a quadratic equation in $r$.

Based on the discussion above, the number of parameters which should be optimized numerically is reduced to three. In the suggested bound, one optimize numerically the parameters $\alpha, \rho, u$ under the following constraints:

$$
0 \leq \rho \leq 1, \quad 0 \leq \alpha<\frac{1}{1-\rho}, \quad 0 \leq \alpha u<\frac{1}{1-\rho}
$$

and the other two parameters ( $\lambda$ and $r$ ) are determined based on (18) and the solution of the above polynomial equation in $r$ (as to tighten the bound (10), where $d=d_{\min }, \cdots, d_{\max }$ ). 


\subsection{Geometrical interpretation of the upper bound (10)}

In [11], we provide a geometric interpretation of the Gallager-type bounds. Based on the discussion there, we conclude that the connection between the Fano-Gallager tilting [11] associated with the function $f$ and the Duman and Salehi tilting measure $\psi$ is

$$
f(y, a)=\psi(y, a)^{\frac{1}{s}} \cdot p_{0}(y, a)^{1-\frac{1}{s}}
$$

where $s \geq 0$. The substitution of (1) and (4) in (24) gives:

$$
\begin{aligned}
\ln \left(\frac{f(y, a)}{p_{0}(y, a)}\right) & =\frac{1}{s} \ln \left(\frac{\psi(y, a)}{p_{0}(y, a)}\right) \\
& =\frac{1-\alpha}{2 s}\left[y^{2}-\gamma a y \cdot \frac{2(1-\alpha u)}{1-\alpha}+a^{2} \gamma^{2}\left(\frac{1-\alpha\left(u^{2}+v^{2}\right)}{1-\alpha}\right)\right]
\end{aligned}
$$

where $\gamma^{2}=\frac{2 E_{s}}{N_{0}}$. It can be verified that if $0<\alpha<1, \alpha\left(u^{2}+v^{2}\right)<1$ and $(u-1)^{2}<\alpha v^{2}$, then the geometrical region which is associated with the Fano-Gallager 1961 bounding technique $[2,11]$ is

$$
\left\{(\underline{y}, \underline{a}) \mid \sum_{i=1}^{n} \ln \left(\frac{f\left(y_{i}, a_{i}\right)}{p_{0}\left(y_{i}, a_{i}\right)}\right) \leq n R\right\} .
$$

Based on (24) and (26), this region can be rewritten in the form

$$
\left\|\underline{y}-\left(\underline{a}, \underline{x}_{0}\right) \cdot \gamma \xi e^{j \phi}\right\|^{2} \leq n R^{\prime}
$$

where $\underline{x}_{0}$ is an $n$-length vector of ' 1 ' (corresponding to the transmitted all-zero codeword), the scaling factor $\xi$ and the rotation angle $\phi$ are related to the parameters of the bound (10) as follows

$$
\xi^{2}=\frac{1-\alpha\left(u^{2}+v^{2}\right)}{1-\alpha}, \quad \phi=\cos ^{-1}\left(\frac{1-\alpha u}{\xi(1-\alpha)}\right), \quad R^{\prime}=\frac{2 s R}{1-\alpha}
$$

where we assume that

$$
0<\alpha<1, \quad \alpha\left(u^{2}+v^{2}\right)<1, \quad(u-1)^{2}<\alpha v^{2} .
$$

These constraints cover all the region where $\xi \geq 0$ and $-\pi \leq \phi<\pi$. Therefore, the spherical region (27) which is associated with the Fano-Gallager 1961 bound (based on the exponential tilting $\psi(\cdot, \cdot)$ in (4) for the generalized second version of Duman and Salehi bounds) coincides with the 
geometrical interpretation of a Gallager-based version of the Divsalar \& Biglieri bound [8, 9]. We conclude that since the constraints on the parameters of the upper bound combined by (10) and (20) are less restrictive than those in (29), then the upper bound here is at least as tight as the Divsalar \& Biglieri bound [8,9] (the former bound may be in fact better than the latter bound). We note that the upper bound (10) involves in its final form a numerical optimization of three parameters (see Section 2.4), which is also the case with the computation of Divsalar \& Biglieri bound. However, the upper bound here does not require integrations, in contrast to the Divsalar \& Biglieri upper bound $[8,9]$, and also in contrast to the generalization of the second version of the Duman and Salehi bounds with the optimal tilting measure $\psi$ (see [10]).

\section{An Example}

We examine here the ensemble performance of the uniformly interleaved repeat-accumulate (RA) codes [3] of rate $-\frac{1}{4}$, where the information block length is $N=1024$ and every information is repeated $q=4$ times (see Fig. 1). The ensemble of codes is assumed to be transmitted through a fully interleaved Rayleigh fading channel with perfect CSI at the receiver. For the calculation of upper bounds on the bit error probability under ML decoding, we rely here on the closed form expression of the average input-output weight distribution of uniformly interleaved RA codes which was derived in [3], which yields that $S_{0}^{\prime}=0$ and

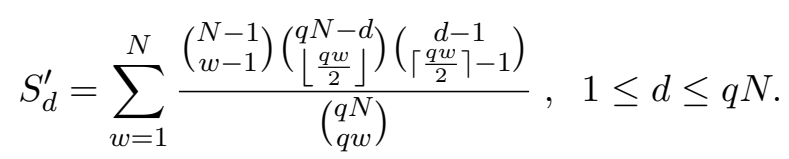

The sequence $\left\{S_{d}^{\prime}\right\}_{d=0}^{q N}$ which is used to calculate upper bounds on the bit error probability under ML decoding for the considered ensemble of RA codes is depicted in Fig. 1(b) in [10].

In Fig. 2, several upper bounds on the bit error probability which apply to the optimal ML decoding are depicted.

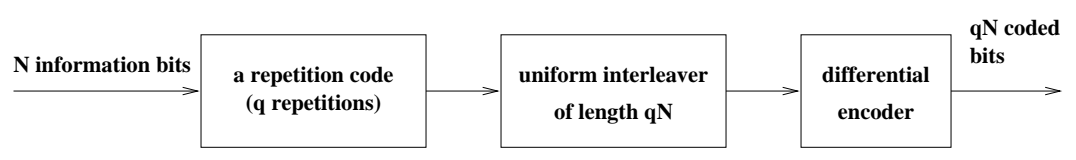

Figure 1: The encoder of RA codes 


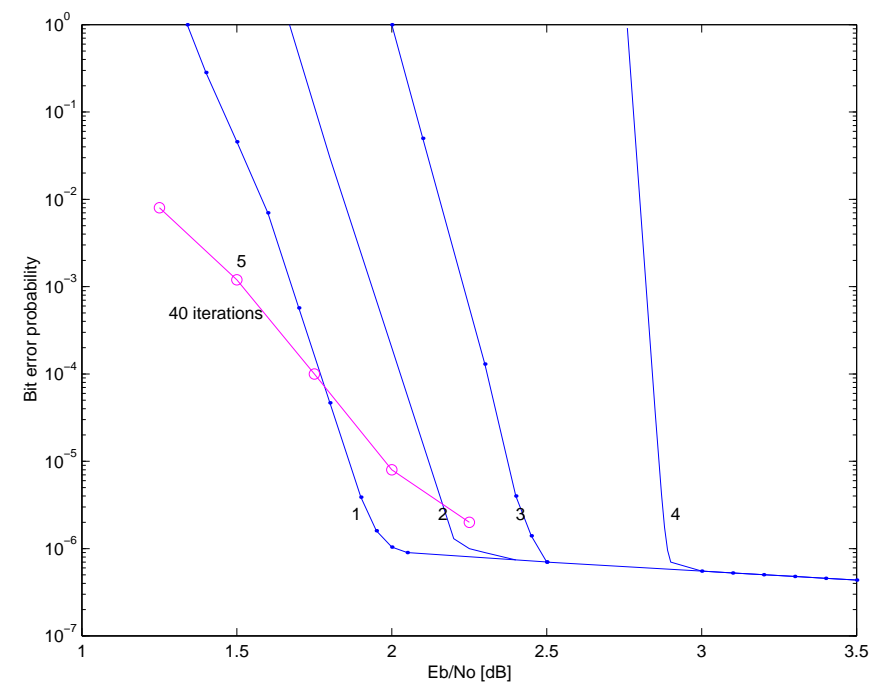

Figure 2: A comparison of upper bounds on the bit error probability of uniformly interleaved RA codes, transmitted over a fully interleaved Rayleigh fading channel. All the upper bounds refer to the optimal ML decoding, and refer to an ensemble of rate $-\frac{1}{4}$. The curves which are depicted in the graph are: 1 - Generalization of the Duman and Salehi bound with the optimal tilting measure, 2 - The upper bound in (10), 3 - generalization of Viterbi \& Viterbi bound, 4 - union bound, 5 computer simulation results of the sum-product iterative decoding algorithm with 40 iterations.

The generalization of the Duman and Salehi bound with the optimal tilting measure (curve 1, see [10]) is the tightest bound among the upper bounds depicted in Fig. 2. The upper bound proposed here which is based on the generalization of the Duman and Salehi bound with the exponential tilting measure in (4) (curve 2) is clearly looser than the former bound which relies on the optimal tilting measure, but is tighter than the generalization of the Viterbi \& Viterbi bound (curve 3) for fully interleaved fading channels. This observation is expected by the arguments put forth in Section 2.2, as the Viterbi \& Viterbi bound was demonstrated as a particular case of the bound (10) proposed here. The three bounds depicted in curves $1-3$, are evidently tighter than the ubiquitous union bound (see curve 4), and are also effective at a portion of the rate region exceeding the cutoff rate, which for the rate $\frac{1}{4}$ equals $\frac{E_{b}}{N_{0}}=2.71 \mathrm{~dB}$. The capacity for rate $\frac{1}{4}$ is attained at $\frac{E_{b}}{N_{0}}=-0.08$ $\mathrm{dB}$. The various upper bounds on the bit error probability (curves 1-4) are also compared here with computer simulation results of the (efficient and sub-optimal) sum-product iterative decoding algorithm with 40 iterations (see curve 5). It is easily realized in Fig. 2 that at a portion of the $\frac{E_{b}}{N_{0}}$ values which correspond to rates above the channel cut-off rate, the improved upper bounds on 
the bit error probability with ML decoding are advantageous over the computer simulation results of the iterative decoding algorithm, which demonstrates the sub-optimality of the latter decoding algorithm. We note here that the tightest upper bound of the generalization of the second version of the Duman and Salehi bound was studied and evaluated for various fading channel models (e.g, Rayleigh and Rician fading channels with or without space diversity) in [10]. This upper bound is subject to numerical integrations and optimizations, while the bounds which are considered here are slightly looser then the former bound, but are expressed in closed form expressions. The reader who is interested in more applications of the former upper bound is referred to [10].

\section{Summary and Conclusions}

We derive here an upper bound on the decoding error probability under ML decoding, which is applicable for binary linear block codes which are transmitted over fully interleaved Rician fading channels with perfect channel side information (CSI) at the receiver. This upper bound is a particular case of the generalization of the second version of Duman and Salehi bounds [10], since the tilting measure $\psi$ is taken in this paper as an exponential tilting measure with respect to the fading $(a)$ and the received vector $(y)$ (rather than the optimal function ' $\psi$ ' which yields an upper bound whose calculation involves numerical integrations, see $[7,10,11,13])$. This bound yields the generalized version of the Viterbi \& Viterbi bound as a particular case (this generalized bound is derived in the Appendix of this paper, and it forms a generalization of the Viterbi \& Viterbi bound [14] which was derived for a binary-input AWGN channel). The bound in this paper also forms a generalization of the first version of the Duman and Salehi bounds, which was derived for a binary-input AWGN channel [12]. It coincides with the geometrical interpretation of the Divsalar \& Biglieri bound $[8,9]$, where this observation is demonstrated by showing that both bounds define the same geometrical region in terms of the Fano-Gallager 1961 bounding terminology [2] (see also [11]). The current bound admits a closed form expression (up to three parameters that should be optimized numerically) for a general fully interleaved Rician fading channel. The upper bound which is derived in this paper does not require integrations, in contrast to a Gallager-based version of the Divsalar \& Biglieri upper bound [8,9], and also in contrast to the generalization of the second version of the Duman and Salehi bounds with the optimal tilting measure $\psi$ (see the DS2 bound in [10]). From this discussion, it is clear that the computational complexity of this bound is reduced as compared to the former two upper bounds (which also involve numerical optimizations of 
parameters in addition to numerical integrations). Sason and Shamai have reported in [10] that the generalization of the second version of the Duman and Salehi bound was efficiently implemented in MATLAB software. However, the bound which was proposed here is evidently less heavy in terms of its computational complexity, with the penalty of a slight reduction in the tightness of the latter bound (as compared to the generalization of the second version of the Duman and Salehi bounds for fully interleaved fading channels [10], which is the tightest reported upper bound under ML decoding). We note that the bound which is derived in this paper (see Sections 2.1 and 2.4) is as tight as the Divsalar \& Biglieri bound, but is simpler in terms of computational complexity (as both bounds involve numerical optimizations of three parameters, but the former bound is given in a closed form expression, while the computation of a Gallager-based version of the Divsalar \& Biglieri bound involves tasks of numerical integrations). The interested reader is referred to [10] for more worked out examples which compare performance bounds of turbo-like codes under ML decoding with computer simulation results of iterative decoding, under the assumption of BPSK modulation and transmission over fully interleaved Rician fading channels with perfect CSI at the receiver. The case of imperfect estimates of the i.i.d. fading samples at the receiver is addressed in $[7,11,16]$.

\section{Appendix: Extension of the Viterbi \& Viterbi upper bound on error probability for fading channels}

Consider a linear $(n, k)$ block code $C$ with code rate $R_{c}=k / n$ and the minimum distance $d_{\text {min }}$, and the maximum distance $d_{\max }$. We consider concatenated codes with interleavers as an $(\mathrm{n}, \mathrm{k})$ block code. Following the derivations in [14], divide the codewords $\mathbf{x}$ with output weight $d$ into subsets $C_{d}, d=0,1,2, \ldots, n$. Cardinality of these sets are $\left|C_{d}\right|=S_{d}$, where $S_{d}$ is the number of codewords of the block code with Hamming weight $d$. Define

$$
P(d)=\operatorname{Pr}\left\{\text { some } \mathbf{x} \in C_{d} \text { is chosen in preference to the all zero codeword }\right\},
$$

then using union bound we obtain (2).

Let the observation samples at the output of channel given the all one sequence $X_{0}$ is transmitted be modeled as:

$$
y_{i}=\gamma a_{i}+n_{i}
$$


where $a_{i}$ are independent fading samples with unit average power, $\gamma=\sqrt{\frac{2 E_{s}}{N_{0}}}$, and $n_{i}$ are zero mean, unit variance Gaussian noise samples. Assume the fading samples are perfectly known at the receiver and let $\underline{a}=\left(a_{1}, a_{2}, \ldots, a_{n}\right)$ be the vector of the independent fading samples. Consider the correlator output for $X_{0}$, as

$$
Y_{0}=\sum_{i=1}^{n} a_{i} y_{i}
$$

and the correlator output for other codewords as

$$
Y_{m}^{(d)}=\sum_{i=1}^{n} a_{i} y_{i} x_{m, i}^{(d)}
$$

where $x_{m, i}^{(d)}$ has negative ones i.e. -1 in $d$ positions and +1 in $(n-d)$ positions. Then we get,

$$
E\left\{Y_{0} \mid X_{0}, \underline{a}\right\}=\gamma \sum_{i=1}^{n} a_{i}^{2},
$$

and

$$
\begin{gathered}
E\left\{Y_{m}^{(d)} \mid X_{0}, \underline{a}\right\}=\gamma \sum_{i=1}^{n} a_{i}^{2} x_{m, i}^{(d)}, \\
\operatorname{Var}\left\{Y_{m}^{(d)} \mid X_{0}, \underline{a}\right\}=\operatorname{Var}\left\{Y_{0} \mid X_{0}\right\}=\sum_{i=1}^{n} a_{i}^{2},
\end{gathered}
$$

Define

$$
Z_{m}^{(d)}=Y_{m}^{(d)}-Y_{0} \cdot\left(\frac{\sum_{i=1}^{n} a_{i}^{2} x_{m, i}^{(d)}}{\sum_{i=1}^{n} a_{i}^{2}}\right)
$$

then we get:

$$
\begin{gathered}
\operatorname{Cov}\left\{Z_{m}^{(d)}, Y_{0} \mid X_{0}, \underline{a}\right\}=0, \\
E\left\{Z_{m}^{(d)} \mid X_{0}, \underline{a}\right\}=0 .
\end{gathered}
$$

Since

$$
\operatorname{Cov}\left\{Y_{0}, Y_{m}^{(d)} \mid X_{0}, \underline{a}\right\}=\sum_{i=1}^{n} a_{i}^{2} x_{m, i}^{(d)}
$$


depends on $m$ (though for the AWGN channel, it depends only on $d$ ) we cannot use Viterbi's independence concept [14] to proceed. So straightforward Viterbi's method will not work for fading. Note that Viterbi started with

$$
E\left\{\left[\sum_{m=1}^{S_{d}} \exp \left(\lambda\left(Y_{m}^{(d)}-Y_{0}\right)\right)\right]^{\rho} \mid X_{0}\right\}
$$

We can write

$$
Y_{m}^{(d)}-Y_{0}=\left[Y_{m}^{(d)}-(1-\alpha) Y_{0}\right]-\alpha Y_{0}
$$

For AWGN, choosing $\alpha=2 d / n$, makes $\left[Y_{m}^{(d)}-(1-\alpha) Y_{0}\right]$ independent of $Y_{0}$. For fading we can optimize $\alpha$ as a parameter. Thus for $0 \leq \rho \leq 1$ we have

$$
\begin{gathered}
P(d) \leq E\left\{\left[\sum_{m=1}^{S_{d}} \exp \left(\lambda\left(Y_{m}^{(d)}-Y_{0}\right)\right)\right]^{\rho} \mid X_{0}\right\} \\
\leq E\left\{\exp \left(-\alpha \lambda \rho Y_{0}\right) \mid X_{0}\right\}\left[\sum_{m=1}^{S_{d}} E\left\{\frac{\exp \left(-\alpha \lambda \rho Y_{0}\right)}{\left.E\left\{\exp \left(-\alpha \lambda \rho Y_{0}\right) \mid X_{0}\right)\right\}} \exp \left(\lambda\left(Y_{m}^{(d)}-(1-\alpha) Y_{0}\right)\right) \mid X_{0}\right\}\right]^{\rho}
\end{gathered}
$$

For AWGN we have $\alpha=2 d / n$, since $Y_{0}$ is independent of $Y_{m}^{(d)}-(1-\alpha) Y_{0}$, so we get

$$
P(d) \leq E\left\{\exp \left(-\alpha \lambda \rho Y_{0}\right) \mid X_{0}\right\}\left[\sum_{m=1}^{S_{d}} E\left\{\exp \left(\lambda\left(Y_{m}^{(d)}-(1-\alpha) Y_{0}\right)\right) \mid X_{0}\right\}\right]^{\rho}
$$

which corresponds to Viterbi's result. However for fading channel we should compute

$$
P(d) \leq\left[E\left\{\exp \left(-\alpha \lambda \rho Y_{0}\right) \mid X_{0}\right\}\right]^{1-\rho}\left[\sum_{m=1}^{S_{d}} E\left\{\exp \left(-\alpha \lambda \rho Y_{0}\right) \exp \left(\lambda\left(Y_{m}^{(d)}-(1-\alpha) Y_{0}\right)\right) \mid X_{0}\right\}\right]^{\rho}
$$

or

$$
P(d) \leq\left[E\left\{\exp \left(-\alpha \lambda \rho Y_{0}\right) \mid X_{0}\right\}\right]^{1-\rho}\left[\sum_{m=1}^{S_{d}} E\left\{\exp \left(\lambda\left(Y_{m}^{(d)}-(1-\alpha(1-\rho)) Y_{0}\right)\right) \mid X_{0}\right\}\right]^{\rho}
$$

where expectations are with respect to both the noise and the fading distributions. If $Y$ is a Gaussian r.v. with mean $\eta$ and variance $\sigma^{2}$ then

$$
E\left\{e^{\beta Y}\right\}=e^{\eta \beta+\frac{1}{2} \sigma^{2} \beta^{2}}
$$


using this result then we have

$$
E\left\{e^{-\alpha \lambda \rho Y_{0}} \mid X_{0}, \mathbf{a}\right\}=\prod_{i=1}^{n} e^{\left(-\alpha \lambda \rho \gamma+\frac{1}{2} \alpha^{2} \lambda^{2} \rho^{2}\right) a_{i}^{2}}
$$

Let $Y=Y_{m}^{(d)}-(1-\alpha(1-\rho)) Y_{0}$ then

$$
\begin{gathered}
\eta=E\left\{Y \mid X_{0}, \mathbf{a}\right\}=\sum_{i=1}^{n} a_{i}^{2}\left[\gamma x_{m, i}^{(d)}-\gamma(1-\alpha(1-\rho))\right] \\
\sigma^{2}=\operatorname{Var}\left\{Y \mid X_{0}, \mathbf{a}\right\}=\sum_{i=1}^{n}\left\{a_{i}^{2}\left(1-2(1-\alpha(1-\rho)) x_{m, i}^{(d)}+(1-\alpha(1-\rho))^{2}\right)\right\} .
\end{gathered}
$$

using the above results we get

$$
E\left\{e^{\lambda\left(Y_{m}^{(d)}-(1-\alpha(1-\rho)) Y_{0}\right)} \mid X_{0}, \mathbf{a}\right\}=e^{\eta \lambda+\frac{1}{2} \sigma^{2} \lambda^{2}}
$$

or

$$
E\left\{e^{\lambda\left(Y_{m}^{(d)}-(1-\alpha(1-\rho)) Y_{0}\right)} \mid X_{0}, \mathbf{a}\right\}=\prod_{i=1}^{n}\left\{e^{a_{i}^{2}\left\{\lambda \gamma\left[x_{m, i}^{(d)}-(1-\alpha(1-\rho))\right]+\frac{1}{2} \lambda^{2}\left[x_{m, i}^{(d)}-(1-\alpha(1-\rho))\right]^{2}\right\}}\right\}
$$

The Rician density function is used to model the density function of independent fading samples. The normalized version of Rician density function $\left(E\left\{a^{2}\right\}=1\right)$ is expressed in (5), which yields:

$$
E\left\{e^{-\beta a^{2}}\right\}=\left(\frac{1+K}{1+K+\beta}\right) e^{-\frac{K \beta}{1+K+\beta}} \quad \text { if } 1+K+\beta>0
$$

where the expectation is with respect to the normalized Rician density function. From (A.3), it can be verified that

$$
E\left\{e^{-\alpha \lambda \rho Y_{0}} \mid X_{0}\right\}=\left(\frac{1+K}{1+K+\beta_{3}}\right)^{n} e^{-\frac{K \beta_{3} n}{1+K+\beta_{3}}}
$$

where

$$
\beta_{3}=\alpha \lambda \rho \gamma-\frac{1}{2} \alpha^{2} \lambda^{2} \rho^{2}
$$

Moreover, it can be shown that

$$
E\left\{e^{Y_{m}^{(d)}-(1-\alpha(1-\rho)) Y_{0}} \mid X_{0}\right\}=\left(\frac{1+K}{1+K+\beta_{1}}\right)^{d} e^{-\frac{K \beta_{1} d}{1+K+\beta_{1}}}\left(\frac{1+K}{1+K+\beta_{2}}\right)^{n-d} e^{-\frac{K \beta_{2}(n-d)}{1+K+\beta_{2}}}
$$


where

$$
\beta_{1}=\lambda \gamma(2-\alpha(1-\rho))-\frac{1}{2} \lambda^{2}(2-\alpha(1-\rho))^{2},
$$

and

$$
\beta_{2}=-\lambda \gamma \alpha(1-\rho)-\frac{1}{2} \lambda^{2} \alpha^{2}(1-\rho)^{2} .
$$

Thus:

$$
\begin{aligned}
P(d) \leq & S_{d}^{\rho}\left(\frac{1+K}{1+K+\beta_{3}}\right)^{n(1-\rho)} e^{-\frac{K \beta_{3} n(1-\rho)}{1+K+\beta_{3}}} \\
& \cdot\left(\frac{1+K}{1+K+\beta_{1}}\right)^{d \rho} e^{-\frac{K \beta_{1} d \rho}{1+K+\beta_{1}}} \\
& \cdot\left(\frac{1+K}{1+K+\beta_{2}}\right)^{(n-d) \rho} e^{-\frac{K \beta_{2}(n-d) \rho}{1+K+\beta_{2}}}
\end{aligned}
$$

Let $\lambda=\mu \gamma, \mu>0$, and $c \triangleq \frac{\gamma^{2}}{2}$ then

$$
\begin{gathered}
\beta_{3}=c\left[1-(1-\alpha \mu \rho)^{2}\right], \\
\beta_{1}=c\left[1-(1-\mu(2-\alpha(1-\rho)))^{2}\right], \\
\beta_{2}=c\left[1-(1+\mu \alpha(1-\rho))^{2}\right] .
\end{gathered}
$$

Let $\delta \triangleq \frac{d}{n}$, and $\operatorname{r}(\delta) \triangleq \frac{\ln S_{d}}{n}$, then

$$
P(d) \leq e^{-n E(c, \delta, K, \rho, \alpha, \mu)},
$$

where

$$
\begin{aligned}
E(c, \delta, K, \rho, \alpha, \mu)= & -\rho \mathrm{r}(\delta)-\rho \delta \ln \left(\frac{1+K}{1+K+\beta_{1}}\right)+\rho \delta\left(\frac{K \beta_{1}}{1+K+\beta_{1}}\right) \\
& -\rho(1-\delta) \ln \left(\frac{1+K}{1+K+\beta_{2}}\right)+\rho(1-\delta)\left(\frac{K \beta_{2}}{1+K+\beta_{2}}\right) \\
& -(1-\rho) \ln \left(\frac{1+K}{1+K+\beta_{3}}\right)+(1-\rho)\left(\frac{K \beta_{3}}{1+K+\beta_{3}}\right)
\end{aligned}
$$


For Rayleigh fading we have $K=0$, thus the exponent reduces to:

$$
\begin{aligned}
E(c, \delta, \rho, \alpha, \mu)= & -\rho \mathrm{r}(\delta)+\rho \delta \ln \left[1+c-c[1-\mu(2-\alpha(1-\rho))]^{2}\right] \\
& +\rho(1-\delta) \ln \left[1+c-c[1+\mu \alpha(1-\rho)]^{2}\right] \\
& +(1-\rho) \ln \left[1+c-c[1-\mu \alpha \rho]^{2}\right]
\end{aligned}
$$

For bit error probability we need to replace $\mathrm{r}(\delta)$ in the above results simply by $\mathrm{r}(\delta) \triangleq \frac{\ln \sum_{w} \frac{w}{k} A_{w, d}}{n}$ where $A_{w, d}$ is the input-output weight coefficient, i.e. the number of codewords of the block code with output weight $d$ associated with an input sequence of weight $w$.

To optimize the parameters, let $\zeta=\mu \alpha$. Taking derivative of exponent with respect to $\mu$ implies that $\beta_{1}=c, \beta_{2}=c\left[1-(1+\zeta(1-\rho))^{2}\right]$, and $\beta_{3}=c\left[1-(1-\zeta \rho)^{2}\right]$. Therefore the bound can be optimized numerically over two parameters $\zeta \geq 0$, and $0 \leq \rho \leq 1$.

For a binary-input AWGN channel, as $K \rightarrow \infty$ the exponent reduces to:

$$
E(c, \delta, \rho, \zeta)=-\rho \mathrm{r}(\delta)+\rho \delta c+\rho(1-\delta) \beta_{2}+(1-\rho) \beta_{3} .
$$

The optimum $\zeta$ is

$$
\zeta=\frac{\delta}{1-\delta+\delta \rho}
$$

For this optimum value, the exponent reduces to $E(c, \delta, \rho)=-\rho \mathrm{r}(\delta)+\frac{c \delta \rho}{1-\delta+\delta \rho}$.

Now, the optimum $\rho$ is $\rho=1$ if $c \geq \frac{\mathrm{r}(\delta)}{\delta(1-\delta)}$. In this case, the exponent corresponds to conventional union bound: $E(c, \delta, \rho=1)=-\mathrm{r}(\delta)+c \delta$.

\section{References}

[1] C. Berrou, A. Glavieux and P. Thitimajshima, "Near Shannon limit error - correcting coding and decoding", Proceedings 1993 IEEE International Conference on Communications (ICC 1993), pp. 1064 1070, Geneva, Switzerland, May 1993.

[2] R. G. Gallager, Low-Density Parity-Check Codes, Cambridge, MA, USA, MIT Press, 1963.

[3] D. Divsalar, H. Jin and R.J. McEliece, "Coding theorems for 'turbo-like' codes", Proceedings 1998 Allerton Conference, Urbana-Chamapain, Illinois, USA, pp. 201-210, September 1998. 
[4] R.J. McEliece, "How to compute weight enumerators for convolutional codes", Communications and Coding, Edited by M. Darnel and B. Honary, pp. 121-141, John Wiley \& Sons Inc., 1998.

[5] I. Sason, E. Telatar and R. Urbanke, "On the asymptotic input-output weight distributions and thresholds of convolutional and turbo-like encoders", IEEE Trans. on Information Theory, vol. 48, no. 12, pp. 3052-3061, December 2002.

[6] D. Burshtein and G. Miller, "Asymptotic enumeration methods for analyzing LDPC codes", presented at the $40^{\text {th }}$ Annual Allerton Conference on Communications, Control and Computing, UrbanaChampaign, Illinois, USA, October 2-4, 2002.

[7] I. Sason, "Upper bounds on the maximum likelihood decoding error probability for block codes and turbo-like codes", Ph.D. dissertation, Department of Electrical Engineering, Technion - Israel Institute of Technology, Haifa, Israel, October 2001. [Online]. Available: http://lthiwww.epfl.ch/ eeigal.

[8] D. Divsalar and E. Biglieri, "Upper bounds to error probabilities of coded systems beyond the cutoff rate", Proceedings 2000 IEEE International Symposium on Information Theory, p. 288, June 2000.

[9] D. Divsalar and E. Biglieri, "Upper bounds to error probabilities of coded systems over AWGN and fading channels", Proceedings 2000 IEEE Global Telecommunications Conference (GLOBECOM'00), pp. 1605-1610, San Francisco, California, USA, 27 November - December 1, 2000.

[10] I. Sason and S. Shamai, "Improved upper bounds on the decoding error probability of block codes over interleaved fading channels, with applications to turbo like codes", IEEE Trans. on Information Theory, vol. 47, no. 6, pp. 2275-2299, September 2001.

[11] S. Shamai and I. Sason, "Variations on the Gallager bounds, connections and applications", IEEE Trans. on Information Theory, vol. 48, no. 12, pp. 3029-3051, December 2002.

[12] T.M. Duman and M. Salehi, "New performance bounds for turbo codes", IEEE Trans. on Communications, vol. 46, no. 6, pp. 717-723, June 1998.

[13] T. M. Duman, "Turbo codes and turbo coded modulation systems: Analysis and performance bounds", Ph.D. dissertation, Department of Electrical and Computers Engineering, Northeastern University, Boston, MA, USA, May 1998.

[14] A.M. Viterbi and A.J. Viterbi, "Improved union bound on linear codes for the binary-input AWGN channel, with application to turbo decoding", Proceedings IEEE Information Theory Workshop, p. 72, San Diego, California, February 1998. 
[15] F. Babich, G. Montorsi and F. Vatta, "On the Viterbi and Viterbi's improved union bounds on turbo codes performance", Proceedings of Second International Symposium on Turbo Codes and Related Topics, Brest, France, pp. 527-531, 4-7 September 2000.

[16] H. Shin and J. H. Lee, "Improved upper bound on the bit error probability of turbo codes for ML decoding with imperfect CSI in a Rayleigh fading channel", Proceedings 2001 IEEE 12th International Symposium on Personal, Indoor and Mobile Radio Communications (PIMRC 2001), San Diego, CA, USA, pp. A.169-A.173, September 2001.

Igal Sason (S'98-M'02) was born in Haifa, Israel, on May 1969. He received the B.Sc. and Ph.D. degrees in electrical engineering from the Technion-Israel Institute of Technology, Haifa, Israel, in 1992 and 2001, respectively.

During 1993-1998, he served in the army as an electrical engineer. During 2001-2003, he has been a Scientific Collaborator in the Faculty of Computer Science and Communications, EPFL- Swiss Federal Institute of Technology, Lausanne, Switzerland. On October 2003, he will join the Department of Electrical Engineering at the Technion as a Senior Lecturer.

Dr. Sason is a co-recipient of the 2003 IEEE Information Theory Society and Communications Society joint paper award. His research interests include information theory and coding theory. He is especially interested in codes on graphs and iterative decoding algorithms, performance bounds of linear codes, and the tradeoff between their performance and complexity.

Shlomo Shamai (Shitz) (S'80-M'82-SM'89-F'94) received the B.Sc., M.Sc., and Ph.D. degrees in electrical engineering from the Technion-Israel Institute of Technology, in 1975, 1981 and 1986 respectively.

During 1975-1985 he was with the Signal Corps Research Labs (Israel Defense Forces) in the capacity of a Senior Research Engineer. Since 1986 he is with the Department of Electrical Engineering, Technion-Israel Institute of Technology, where he is now the William Fondiller Professor of Telecommunications. His research interests include topics in information theory and digital and analog communications. He is especially interested in theoretical limits in communication with practical constraints, multi-user information theory and spread spectrum systems, information- 
theoretic models for multiuser cellular radio systems and magnetic recording, channel coding, combined modulation and coding, turbo coding and iterative detection/decoding algorithms, digital spectrally efficient modulation methods employing coherent and noncoherent detection and digital communication in optical channels.

Dr. Shamai (Shitz) was the recipient of the 1999 van der Pol Gold Medal of the Union Radio Scientifique Internationale (URSI) and a co-recipient of the 2000 IEEE Donald G. Fink Prize Paper Award. He also received the 2000 Technion Henry Taub Prize for Excellence in Research, and he is also a co-recipient of the IEEE Communication Society and Information Theory joint paper award for 2003. He has served as Associate Editor for the Shannon Theory of the IEEE TRAnsactions on Information Theory, and also served six years on the Board of Governors of the IEEE Information Theory Society, to which he was reelected in 2002.

Dariush Divsalar (S'76-M'78-SM'90-F'97) Dr. Dariush Divsalar received his Ph.D. degree in Electrical Engineering from UCLA in 1978. Since then, he has been with Jet Propulsion Laboratory, California Institute of Technology, where he is a senior research scientist. At JPL, he was working on developing state-of-the-art technology for advanced deep space communications systems and future NASA space exploration and mobile satellite communications.

In 2001 he joined Sequoia Communications as Director of Algorithm Development. At Sequoia Communications, in addition to management duties, he is working in developing innovative low complexity algorithms to improve baseband WCDMA receiver.

His areas of interest are turbo coding, digital modulation, CDMA, and multiuser interference cancellation methods. During 1986-1996, he has taught digital communications, spread spectrum communications, and coding at UCLA. During 1997-2001, he has taught graduate courses in digital communications at Caltech. He has published over 120 papers, co-authored a book entitled "An Introduction to Trellis Coded Modulation with Applications" (MacMillan, 1991), and holds eight U.S. patents on the above areas. He is the co-recipient of the 1986 Prize Paper Award in Communications for the IEEE Transactions on Vehicular Technology. Recently one of his papers has been selected as one of the key research papers published by the IEEE Communications Society during the past five decades. Dr. Divsalar received over 20 NASA Tech Brief awards and a NASA Exceptional Engineering Achievement Medal in 1996. He served as Editor, and Area Editor in Coding and Communication Theory for the IEEE Transactions on Communications from 1989 to 1996. Dr. Divsalar is a Fellow of IEEE. 